\title{
The impact of poor sleep on cognitive and activities of daily living after traumatic brain injury: a review
}

\section{Short title:}

Sleep and everyday activities after TBI

\section{Authors :}

Catherine Duclos, B.A., ${ }^{1-2}$ Marie-Pascale Beauregard, M. Sc. .O.T. ${ }^{3}$ Carolina Bottari, Ph. D., O.T. ${ }^{3,6}$ Marie-Christine Ouellet, Ph.D., ${ }^{4-5}$ Nadia Gosselin, Ph.D., ${ }^{1,7}$

\section{Affiliations :}

1. Center for Advanced Research in Sleep Medicine, Hôpital du Sacré-Coeur de Montréal, Montreal, Canada

2. Department of Psychiatry, Université de Montréal, Montreal, Canada

3. Occupational Therapy program, School of Rehabilitation, Université de Montréal, Montreal, Canada

4. École de psychologie, Université Laval, Quebec, Canada

5. Institut de réadaptation en déficience physique de Québec, Quebec, Canada

6. Centre for Interdisciplinary Research in Rehabilitation of Greater Montreal, Montreal, Canada

7. Department of Psychology, Université de Montréal, Montreal, Canada

Australian Occupational Therapy Journal

\section{Address for correspondence:}

Nadia Gosselin, Ph.D.

Center for Advanced Research in Sleep Medicine

Hôpital du Sacré-Cœur de Montréal

5400 boul. Gouin Ouest, local E-0330

Montréal, Québec

H4J 1 C5

Canada

Tel: 514-338-2222 ext. 7717

Fax: 514-338-3893

nadia.gosselin@umontreal.ca 


\section{Abstract}

Background: Patients frequently report sleep disruptions or insomnia during their hospital stay, particularly after a traumatic brain injury (TBI). The consequences of these sleep disturbances on everyday activities are not well documented and are therefore not considered in the evaluation of independence in activities of daily living (ADLs). The goal of this narrative review is to explore the consequences of poor sleep quality on cognition and ADLs in the acute and subacute stages of a moderate and severe TBI, when patients are in acute care or in-patient rehabilitation.

Methods: We will present an overview of normal sleep and its role in cognitive functioning, and then present the findings of studies that have investigated sleep characteristics in hospital settings and the consequences of sleep disturbances on ADLs.

Results: During hospitalisation, TBI patients present severe sleep disturbances such as insomnia and sleep fragmentation, which are probably influenced by both the medical condition and the hospital or rehabilitation environment. Sleep disruption is associated with several cognitive deficits, including attention, memory and executive function impairments. Poor quality and/or insufficient quantity of sleep in acute TBI probably affect general functioning and ADLs calling for these cognitive functions.

Conclusions and significance: The cognitive impairments present following TBI are probably exacerbated by poor sleep quality and sleep deprivation during hospitalisation, which in turn impact ADLs among this population. Healthcare personnel should further consider sleep disturbances among people with TBI and a sleep protocol should be established.

Keywords: traumatic brain injury, sleep, activities of daily living, cognition, critical care 


\section{Introduction}

Every year in the United States, at least 1.4 million individuals suffer a traumatic brain injury (TBI), and the most high-risk individuals are young adults, particularly men, aged 15 to 24 (Langlois-Oman, Kraus, Zaloshnja \& Miller, 2011). In 23 European countries, incidence of hospital admissions for traumatic brain injury are estimated at 235 per 100,000 people (Tagliaferri, Compagnone, Korsic, Servadei \& Kraus, 2006), while incidence rates reach 350 and 325 per 100,000 in Brazil and South Africa, respectively (Roozenbeek, Maas \& Menon, 2013). In Australia, a rate of 107 TBI-related hospital stays per 100,000 people was reported in 20042005, peaking at 300 per 100,000 among 15- to 24-year-olds (Australian Institute of Health and Welfare, 2007). Occupational therapy interventions in acute care consist of evaluating the repercussions of physical, cognitive and behavioural deficits on independence in everyday activities, and their link with decisions about discharge destination as well as the patient's potential for rehabilitation. Severe sleep-wake cycle disturbances documented in acute and subacute TBI (Duclos et al., 2013; Makley et al., 2009; Nakase-Richardson et al., 2013) probably influence cognitive functioning, particularly learning ability, which by extension impact the patient's ability to demonstrate optimal levels of functioning in everyday activities. Surprisingly, few studies have examined sleep difficulties and their impact on activities of daily living (ADL) among patients with acute TBI. Consequently, the influence of sleep on general functioning is often not considered when making clinical recommendations on the basis of ADL evaluation results. The aim of this narrative review is to explore the impact of poor sleep quality and sleep deprivation on cognition and ADLs among individuals with moderate and severe TBI, primarily 
during acute hospitalisation and early inpatient rehabilitation following TBI. We will first present an overview of normal sleep architecture and its role in cognitive functioning. We will then present the findings of studies that have investigated sleep characteristics in hospital settings and the consequences of sleep disturbances on ADLs. Finally, we will describe protocols aimed at improving sleep-wake cycles and the specific role of occupational therapists in their implementation.

\section{Introduction to normal sleep}

Sleep is composed of slow-wave sleep stages and of the paradoxical sleep stage (see Figure 1 for a schematic representation of a normal night of sleep). Slow-wave sleep is divided into three stages, namely stages $\mathrm{N} 1$ and N2, which are light sleep, and N3, also known as stages 3 and 4, or deep sleep (Carskadon \& Dement, 2011; Iber, Ancoli-Israel, \& Quan, 2007; Roehrs, 2005). These three stages are also referred to as non-rapid eye movement (NREM) sleep. Paradoxical sleep, also known as rapid eye movement (REM) sleep, has a similar electroencephalographic (EEG) activity as wakefulness and is characterised by muscle atonia and marked eye movements. Paradoxical sleep is the primary stage during which dreaming occurs. In the course of one night, the sleeper alternates between the various sleep stages in cycles of 90 to 100 minutes. There are on average four to six cycles per night. At the beginning of the night, stage $\mathrm{N} 3$ is the most present of all sleep stages, while stage N2 and REM sleep are the most prevalent stages towards the end of the night.

Whereas adolescents (ages 13-17) generally require 9 hours of sleep, adults sleep an average of 7-8 hours per night (Foley, Ancoli-Israel, Britz, \& Walsh, 2004; Mindell, Owens \& 
Carskadon, 1999). Total sleep time decreases with age, whereas the quantity of light sleep increases progressively (Carrier, Monk, Buysse, \& Kupfer, 1997; Ohayon, Carskadon, Guilleminault, \& Vitiello, 2004). It has also been shown that the decline of deep sleep begins around ages 36-50 (Van Cauter, Leproult, \& Plat, 2000). Sleep can be objectively and quantitatively measured by polysomnography (PSG), which minimally includes electroencephalography, electrooculography and electromyography in order to identify each sleep stage. Aside from PSG, other methods are used to evaluate sleep quality, such as actigraphy, an accelerometer that measures movement and is used to monitor the rest-activity cycle, supervision by the nursing staff (when studies occur in hospital settings), and questionnaires.

\section{Role of sleep in cognition}

It is well established that sleep deprivation affects cognitive performance and this is not only true for acute total sleep deprivation, but also for chronic and partial sleep loss (Goel, Rao, Durmer, \& Dinges, 2009). Among the cognitive domains that are simultaneously most affected by poor sleep or sleep loss and most important for ADLs, we find attention, memory, and executive functions (Chee \& Choo, 2004; Choo, Lee, Venkatraman, Sheu, \& Chee, 2005;

Diekelman \& Born, 2010; Dinges, 1992; Goel, Rao, Durmer, \& Dinges, 2009; Harrison \& Horne, 2000; Jones \& Harrison, 2001; Mu et al., 2005; Tomasi et al, 2009; Walker \& Stickgold, 2006). In this section, we will briefly describe the influence of poor sleep or sleep loss on these specific cognitive domains, within a healthy population. 
Attention

Attention is generally defined by its different components, which include vigilance, sustained attention, selective attention, alternating attention, and divided attention (Sohlberg \& Mateer, 1987). Most studies have documented the influence of sleep deprivation on vigilance and sustained attention. It has been observed that sleep deprivation leads to behavioural changes characterised by a general slowing of reaction times, an increased number of errors (omission and commission) on tasks requiring detection of randomly occurring stimuli, and an increased time-on-task effect, which means that performance worsens across the course of a cognitive task (see Lim \& Dinges, 2008 for a review). These behavioural effects of sleep deprivation are not only observed for acute and complete sleep deprivation, but are also observed following partial sleep deprivation. For example, in a study where sleep was restricted to three hours per night for seven days, performance on the vigilance task was affected, with subjects presenting a high rate of omissions when compared to a non-sleep deprived group (Fafrowicz et al., 2010).

\section{Memory}

Two types of memory were found to be very sensitive to poor sleep, namely episodic memory, which refers to the capacity to store and retrieve memories that are associated to a specific time and place, and procedural memory, which enables retention of learned connections between stimuli and responses (Tulving, 1983).

Episodic memory is affected by poor sleep in two ways. Firstly, poor sleep impedes learning of verbal and non-verbal material if learning is preceded by a night of poor sleep (i.e. learning after the night of poor sleep). This has been observed among students, since the 
consequences of sleep deprivation among this chronically sleep deprived population include learning deficits and inferior academic performances (Curcio, Ferrara, \& De Gennaro, 2006). Among healthy subjects and individuals presenting insomnia, a link between total sleep deprivation preceding learning and the presence of memory encoding deficits has also been observed (Fortier-Brochu, Beaulieu-Bonneau, Ivers, \& Morin, 2012; Yoo, Hu, Gujar, Jolesz, \& Walker, 2007). Secondly, poor sleep also impedes learning if learning is followed by a night of poor sleep (i.e. learning prior to a night of poor sleep). In fact, several studies showed the crucial role of sleep in memory consolidation and cerebral plasticity (Atienza, Cantero, \& Stickgold, 2004; Smith, 1996; Walker, 2004; Walker \& Stickgold, 2006; Walker, Stickgold, Gaab, \& Schlaug, 2005; Wilson, 2002). As an example, one study showed that performance on a visual discrimination task was maximally improved 48-96 hours after initial training, even without practice, but only if subjects could sleep within 30 hours of training (Stickgold, James, \& Hobson, 2000). Another study, using learning of synthetic speech, showed that word identification accuracy on the post-test, which took place 12 hours after pre-test, improved by $18.7 \pm 1.6$ percentage points if the 12-hour interval included sleep, whereas it improved by only $10.1 \pm 2.0$ percentage points when no sleep was present between pre-test and post-test. Overall, this study showed that speech recognition performance immediately following training was significantly improved, that it subsequently degraded over the span of a day's retention interval, but completely recovered following sleep (Fenn, Nusbaum, \& Margolish, 2003).

As for procedural memory, several studies have shown that performance is improved when a post-learning sleep period is present (Albouy et al., 2013; Barakat et al., 2012; Fischer, 
Hallschmid, Elsner, \& Born, 2002; Walker, Brakefield, Morgan, Hobson, \& Stickgold, 2002). Indeed, studies have demonstrated that a sleep episode following a period of practice of given motor sequences increases the speed of performance by $20 \%$ and $33.5 \%$, and reduces the error by $30.1 \%$ on the specific motor sequence learned (Fischer, Hallschmid, Elsner, \& Born, 2002).

\section{Executive functions}

Executive functions represent an ensemble of cognitive processes involved in goaloriented behaviour, including abilities related to planning, organisation, problem-solving, inhibition, working memory, judgment, flexibility of thought, and verbal fluency (Alvarez \& Emory, 2006). They enable one to plan, to coordinate a complete series of actions while considering alternatives, to control and update actions when necessary, and to eliminate elements of distraction while paying attention to the task at hand (inhibition) (Welsh \& Pennington, 1988; Welsh, Pennington, \& Groisser, 1991).

Numerous studies have investigated the impact of poor sleep on executive functions, particularly with regards to judgment, perseveration, impulsivity of behaviour, inhibition of distracting elements, planning of tasks, and word generation (for reviews, see Goel, Rao, Durmer, \& Dinges, 2009; Harrison \& Horne, 2000; Jones \& Harrison, 2001; Killgore, 2010; Reynolds \& Banks, 2010). These studies have shown that sleep deprivation does not increase impulsivity among healthy subjects (Acheson, Richards, \& de Wit, 2007; Sagaspe, Charles, Taillard, Bioulac, \& Philip, 2003). However, according to studies carried out among people suffering from sleep apnea, partial sleep deprivation leads to perseveration and to a decline in mental flexibility during tasks. This affects both the ability to adequately use cognitive functions 
in order to have good judgement, and the performance on tasks of verbal fluency and planning (Bedard, Montplaisir, Malo, Richer, \& Rouleau, 1993; Bedard, Montplaisir, Richer, Rouleau, \& Malo, 1991; Olsen, Lawrence, Bottarini, \& Pises, 1977). Though no studies have specifically examined this in TBI, we can hypothesise that individuals with neurological vulnerability, such as those with moderate and severe TBI, would be more sensitive to the impact of sleep loss on executive functions.

\section{Sleep in hospital settings}

Sleep deprivation is one of the most frequent complaints among hospitalised individuals, particularly in the intensive care unit (ICU), where $61 \%$ of people mention suffering from sleep deprivation (Simini, 1999). According to studies performed with non-TBI ICU patients, sleep disturbance is the second most stressful aspect of hospitalisation (Freedman, Gazendam, Levan, Pack, \& Schwab, 2001; Nelson et al., 2001; Richards \& Bairnsfather, 1988). PSG studies showed that sleep is lighter and more fragmented in critically ill patients than among the general population (Broughton \& Baron, 1978; Beecroft et al., 2008; Cooper et al., 2000; Friese et al., 2007; Gabor et al., 2003; Gehlbach et al., 2012; Kavey \& Ahshuler, 1979). In fact, stage N1, which usually represents less than $5 \%$ of the night, accounts for approximately $60 \%$ of the sleep of ICU patients. As for stage N2, contradictory results are reported: certain studies report normal or higher proportions, while others report a decreased proportion. (Friese et al., 2007; Gabor et al., 2003) However, a significant reduction is clearly present for stage N3, while paradoxical sleep is often significantly reduced or absent (Friese et al. 2007; Gabor et al., 2003; Gehlbach et al., 2012; Kavey \& Ahshuler, 2003). 
More recently, sleep in hospital and inpatient rehabilitation settings was specifically investigated among patients with TBI (Duclos et al., 2013; Makley et al., 2009; Makley et al., 2008; Nakase-Richardson et al., 2013). A recent study using actigraphy in a hospital setting has shown that in the acute phase of TBI, patients experience rest-activity cycle disturbances, which globally improve over time (Duclos et al., 2013). In fact, among the 16 patients studied, only 3 $(18.8 \%)$ had a consolidated rest-activity cycle while in ICU. This is defined as sustained periods of activity during the day and sustained periods of rest during the night. Overall, this study showed that of all days of actigraphy recording in the ICU, only $28.6 \%$ days showed a consolidated rest-activity cycle, whereas this proportion increased to $61.1 \%$ on the regular units. In the subacute phase of TBI, a study carried out using actigraphy in a rehabilitation unit showed that sleep-wake cycle was altered in $68 \%$ of patients (Makley et al., 2009). These patients with sleep-wake cycle disturbances had longer stays in both acute and rehabilitation settings, implying that sleep-wake disturbances may be associated with more severe injury (Makley et al., 2008). Another study showed that, based on item 1 of the Delirium Rating Scale-revised-98 (Trzepacz et al., 2001), mild to severe sleep disturbances were present among $84 \%$ of TBI patients (mainly severe) upon admission to a rehabilitation hospital, and persisted for $66 \%$ of patients one month post-injury. Results also showed that the presence of such sleep disturbances at one month postinjury was a significant predictor of the duration of post-traumatic amnesia (Nakase-Richardson et al., 2013).

Many factors may account for the poor sleep of hospitalised patients, particularly in the ICU environment. Indeed, several sources of noise are present, including alarms and 
conversations among employees, preventing patients from sleeping well (Freedman, Gazendam, Levan, Pack, \& Schwab, 2001; Freedman, Kotzer, \& Schwab, 1999; Friese et al., 2007; Gabor et al., 2003; Hilton, 1976; Persson Waye, Elmenhorst, Croy, \& Pedersen, 2013; Richardson, Allsop, Coghill, \& Turncock, 2007) In fact, Cohen et al. (1992) found that difficulty initiating and maintaining sleep was highly reported (81.2\%) in hospitalised TBI patients, and that $36 \%$ of patients reporting these sleep disturbances identified the hospital environment as an important causal factor. Receiving 24-hour care also makes sleep more difficult (Freedman, Kotzer, \& Schwab, 1999; Tamburri, DiBrienza, Zozula, \& Redeker, 2004). Furthermore, the loss of light/dark circadian cues due to constant lighting, as well as being bedridden and in a constant horizontal posture, are all factors that promote daytime sleep (Gabor et al., 2003). In fact, an abnormal distribution of sleep over a 24-hour period, marked by a proportion of daytime sleep around $50 \%$, was observed in non-TBI critically ill patients (Freedman, Gazendam, Levan, Pack, \& Schwab, 2001). Moreover, several drugs used as sedatives or analgesics, such as benzodiazepines, opiates, anticonvulsants and antipsychotics, influence sleep characteristics (Borbely, Mattmann, Loepfe, Strauch, \& Lehmann, 1985; Bourne \& Mills, 2004; Cronin, Keifer, King, \& Bixler, 2001; Gimenez et al., 2007; Wilson \& Argyropoulos, 2005).

Overall, $80 \%$ of patients presenting sleep problems report feeling fatigued, which may have a significant impact on ADLs (Ouellet, Beaulien-Bonneau, \& Morin, 2006). Other factors specific to patients with TBI may also contribute to sleep disturbances in hospital and in-patient rehabilitation settings. Recent studies indicate that $95 \%$ of people in the acute phase of moderate to severe TBI have an abnormally low rate of hypocretin-1, a neuropeptide involved in the 
waking state (Baumann et al., 2005; Baumann, Werth, Stocker, Ludwig, \& Bassetti, 2007). This abnormal rate is associated with fatigue and daytime sleepiness, and could reflect hypothalamic damage. Others have suggested that patients who have suffered TBI and present metabolic, endocrine and neurological dysfunctions (i.e. cerebral lesions) run a higher risk of presenting a poorer sleep quality (Breed, Flanagan, \& Watson, 2004). One study carried out at least one year post-injury found an association between neuroendocrine abnormalities and fatigue after TBI, showing that at least one pituitary axis was abnormal among $90 \%$ of TBI patients. More specifically, an association between higher growth hormone levels and greater fatigue was found (Bushnik, Engaldner, \& Katznelson, 2007).

Other variables that might be associated with increased sleep disturbances are pre and postTBI psychiatric and psychological factors. Sleep disturbances can be related to the presence of depression and anxiety, which are both highly prevalent after TBI (Ouellet, Beaulieu-Bonneau, \& Morin, 2006; Parcell, Ponsford, Rajaratnem, \& Redman, 2006). Rao and colleagues (2008) found that insomnia in the acute period following TBI (within 3 months) was closely tied to the appearance of an anxiety disorder. Furthermore, people with TBI can suffer from acute posttraumatic stress disorder, a mental disorder that can manifest itself following a psychologically traumatising event, and can lead to an alteration of sleep and a decrease of slow-wave sleep (American Psychiatric Association, 2013; Bryant, Marosszeky, Crooks, \& Gurka, 2000; Germain, 2013; Yetkin, Aydin, \& Ozgen, 2010).

Changes in life habits and routines may also play a role in the development of sleep disturbance, although still little research has addressed these factors. For example, due to severe, 
persistent and debilitating fatigue, TBI survivors are prone to sleep or stay in bed for large amounts of time either because they feel an increased need for sleep, or rest, or because they lack activities (e.g. routine of getting up for work) (Ouellet, Beaulieu-Bonneau, \& Morin, 2006). This may be particularly true in the acute phase after the accident, when rest is intuitively thought to promote recovery. Unfortunately though, excessive time spent in bed, sleeping or resting, may actually contribute to creating problems in nocturnal sleep by affecting the macrostructure of night-time sleep (Morin, 1993).

\section{Impact of poor sleep on functional outcome}

Poor sleep or sleep loss can affect ADLs and other functional measures evaluated by occupational therapists. Associations between sleep-wake disturbances and general functioning or daily activities have been investigated among TBI patients (Duclos et al., 2013; Worthington \& Melia, 2006). In the study by Duclos and colleagues (2013), absence of a 24-hour sleep-wake cycle during the hospital stay (mean delay of actigraphy start after TBI: $18.0 \pm 13.3$ days) was associated with lower functioning, as measured with the Disability Rating Scale (Rappaport, Hall, Hopkins, Belleza, \& Cope, 1982) and persistent post-traumatic amnesia at hospital discharge in patients with moderate to severe TBI. In rehabilitation centers, Worthington and Melia found that aggressions (related to staff trying to get the person up in the morning), missing out on opportunities for orientation and hygiene programmes in the morning, failing to attend medical appointments and an inability to stay awake during activities were associated with sleep and arousal disturbances (Worthington \& Melia, 2006). Considering the role of sleep in cognition, poor sleep can also affect a patient's results on tests of cognitive screening that are 
often performed in the context of the occupational therapist's evaluation, such as the "Minimental state examination"(MMSE), the Cognitive Assessment Scale for the Elderly (CASE), as well as results on tests used to evaluate driving capacities (e.g.: "Trail making" A et B, "Motorfree visual perception test"(MVPT)) (Colarusso \& Hammill, 2003; Folstein, Folstein, \& McHugh, 1975; Geneau, 1996; Lezak, Howieson, \& Loring, 2004). According to what has been previously reported regarding the impact of sleep on cognition, deficits presented by patients hospitalised secondary to a TBI on these tests could possibly be, at least in part, attenuated if they had better sleep.

Other studies have been carried out among healthy subjects, as well as among subjects suffering from sleep disorders, and poor sleep quality and/or sleep deprivation have consistently been shown to be significantly associated with general functioning and ADL. More specifically, according to Weaver et al. (1997), the activities most thought to be influenced by disrupted sleep and excessive daytime sleepiness are activities that provide minimal external stimulation and that unmask or possibly increase sleepiness such as driving or passive vigilance. Activities that are considered in the Functional Outcome Sleep Questionnaire (FOSQ) they developed to examine the repercussions of excessive daytime sleepiness include: taking care of financial affairs and paperwork, finishing a meal, maintaining a phone conversation, watching television, working on a hobby, remembering things and concentrating on things. Several studies among various populations (e.g. sleep apnea, narcolepsy, non-restorative sleep, pulmonary fibrosis, heart failure) have used the questionnaire developed by Weaver and found that links between disrupted sleep, excessive daytime sleepiness and poorer performance in these activities 
(Banhiran et al., 2012; Carmona-Bernal et al., 2008; Chasens, Sereika, Houze, \& Strollon, 2011; Mermigkis et al., 2013; Riegel et al., 2012; Su, Liu, Panjapornpon, Andrews, \& FoldvarySchaefer, 2012; Teixeira, Faccenda, \& Douglas, 2004; Weaver 2001; Zhang et al., 2013).

In studies carried out using the SF-36 questionnaire, which assesses functional health and well-being through the evaluation of several health domains, physical and social functioning, as well as mental health, functional health and well-being were more affected in individuals with sleep disorders than in individuals who slept normally (Lee et al., 2009; Ware, Snow, Kosinski, \& Gandek, 1993). Accordingly, it has been shown that subjects suffering from insomnia had a poor quality of life, both physically and mentally, and presented a reduced productivity at work as well as alterations of their ADLs (Bolge, Doan, Kannan, \& Baran, 2009). More recently, studies performed among elderly subjects have shown that poor sleep and daytime sleepiness predict poorer functional recovery rates during inpatient rehabilitation, as well as greater functional decline (Frohnhofen, Popp, Frohnhofen, \& Fulda, 2013; Spira et al., 2012).

The importance of ADL evaluations in acute TBI patients cannot be underestimated as they are generally used to recommend discharge needs or rehabilitation potential. When significant sleep deprivation is present, results obtained with ADL measures could reflect the negative effects of sleep deprivation and therefore inadequately influence clinical decisions. Hence, occupational therapy evaluations of ADL ability would need to nuance test results when sleep deprivation is known to have occurred during the night prior to these evaluations. 


\section{Protocols to improve sleep in hospital and rehabilitation centers}

\section{Modifying the environment to improve sleep}

Considering that some factors impeding good sleep quality and duration can be modified, the following section will present protocols aimed at improving sleep-wake cycles, as well as the specific role of occupational therapists in their implementation. Environment is a major cause of sleep disturbances in hospital settings. Fortunately, some environmental factors can be modified. It has been shown that noise is among the factors that interfere the most with sleep in the hospital (Cohen, Oksenberg, Snir, Stern, \& Grosswasser, 1992; Freedman, Gazendam, Levan, Pack, \& Schwab, 2001). Having patients use ear plugs and minimizing staff interventions during periods of napping or during the night would enable patients to have more restorative sleep (Richardson, Allsop, Coghill, \& Turncock, 2007; Topf, 1992; Topf \& Davis, 1993). These methods have been tested among ICU patients and improved sleep was observed (Le Guen, Nicolas-Robin, Lebard, Arnulf, \& Langeron, 2014; Richardson,Allstop, Coghill, \& Turncock, 2007;Wallace, Robins, Alvord, \& Walker, 1999).

Sleep and wake states can also greatly benefit from light during the day and darkness during the night. In fact, the main circadian biological clock, located in the hypothalamus, is normally synchronized to the environmental 24-hour day, mainly by exposure to the light-dark cycle (Czeisler \& Gooley, 2007; Dumont \& Beaulieu, 2007; Takahashi \& Zatz, 1982; Moore, 1997). The sleep-wake cycle is dependent on this circadian biological clock. Light therapy has been shown to be beneficial in synchronizing the circadian clock, improving sleep quality, mood and cognitive performance, (see Munch \& Bromundt, 2012 for a review) and has also been 
found effective in improving sleep and cognitive function among the institutionalised elderly, with or without dementia (Riemersma-van der Lek et al., 2008; Sloane et al., 2007; Van Someren, Kessler, Mirmiran, \& Swaab, 1997). More recently, blue light therapy was show to significantly improve fatigue and drowsiness among chronic TBI patients who reported sleep and wake disturbances (Sinclair, Ponsford, Taffe, Lockley, \& Rajaratnam, 2013). Based on these findings, we could hypothesize that light therapy during acute and post-acute TBI could favor more robust sleep-wake rhythms, though this has not been formally investigated.

\section{Naps to improve cognitive functioning}

It has been shown that a nap of 20 to 30 minutes can reduce fatigue and increase cognitive performances for several hours among healthy subjects (Lovato \& Lack, 2010). The early afternoon is the time of day during which a nap is most beneficial. A protocol comprising the addition of a daily rest period of approximately 30 minutes following lunch could be established in order to further preserve the cognitive functions of TBI patients. To date, no study investigating the effects of naps in a neurological population has been performed and the potential benefits of naps on cognitive functioning and ADL is not known. It is thus recommended that a case-by-case approach, with constant monitoring on the efficacy and negative consequences of various durations and timings of daytime naps, be used. However, considering the important sleep loss that most patients experience during their hospital stay, professionals such as occupational therapists, may be encouraged to interchange, when possible, treatment sessions between patients when a scheduled patient is taking a nap. It could also be important to ensure that patients are allowed to have rest periods between various interventions. 
Most behavioural sleep medicine experts agree that late naps (i.e. taken later than 3:00 PM) may interfere with nighttime sleep (Morin, 2004). Although patients feeling either fatigued or sleepy may want to take a nap after the evening meal, occupational therapists, in collaboration with specialists in recreational activities and/or nurses may contribute to finding alternative activities in order for patients to maintain alertness before bed.

\section{Interventions to improve insomnia}

For patients suffering from insomnia, the implementation of behavioural recommendations such as Stimulus Control or Sleep Restriction (or restriction of time in bed) may be useful (Ouellet \& Morin, 2004; Ouellet \& Morin, 2007). These procedures have a very large evidence-base in the general population for primary insomnia, and are increasingly disseminated in populations with diverse health conditions by non-sleep specialists (Manber et al., 2012). The goal of Sleep Restriction is to consolidate sleep and promote deeper and more continuous sleep through the night by limiting the time spent in bed to the actual sleep time. A sleep window is prescribed and kept consistent for at least 1-2 weeks at first, then is adjusted (increased or decreased) depending on sleep efficiency. Stimulus Control consists of a set of instructions (e.g. keep 1 hour before bed to relax, reserve bed and bedroom for sleep only, go to bed only when sleepy, limit daytime napping, get out of bed if unable to sleep after 15-20 minutes). Largely based on self-management principles, these techniques could nonetheless be used with hospitalised patients if healthcare professionals supply a minimum of structure and guidance. In an acute care or rehabilitation setting, occupational therapists could be instrumental to the implementation of such behavioural interventions for sleep. For example, occupational 
therapists could assist patients in finding appropriate activities in order to maintain a prescribed sleep window and follow Stimulus Control instructions. The following could be implemented by occupational therapists: activities in the evening to counteract sleepiness or the habit of going to bed too early (in line with Sleep Restriction); activities to promote a smooth transition between waking activities and sleep and activities in the morning to avoid staying in bed too long and to increase the motivation for getting out of bed and starting the day (in line with Stimulus Control recommendations); activities during the night to distract from worrying, rumination or intrusive thoughts (in line with cognitive and relaxation-based techniques); activities to avoid smoking before bedtime or during nocturnal awakenings (in line with Sleep Hygiene education); and activities to promote rest during the day without necessarily sleeping (napping), especially late during the day (in line with Stimulus Control recommendations).

The support of nurse colleagues and other professionals (e.g. educators, recreational activities specialists) would be critical to the application of, and adherence to, the various protocols to improve sleep. As such, occupational therapists could have an influential role in raising awareness among the clinical personnel about the impacts of poor sleep and its consequences on the ADLs of people with TBI. The applicability and efficacy of these abovementioned interventions nevertheless need to be investigated among this clinical population and setting. In the meantime, caution and close follow-ups are warranted when any type of intervention is initiated. 


\section{Limits}

Though the focus of this review was to discuss the impact of sleep on cognition and activities of daily living, we did not discuss the effects of medications, particularly sedatives and analgesics, on sleep. Such medications are often administered to TBI patients in acute care and could be responsible, at least in part, for modifications in sleep and wake.

\section{Conclusions}

This literature review has shown that TBI causes changes in sleep that have repercussions on everyday activities. Lack or loss of sleep has a direct impact on cognition, which in turn has a direct impact on independence in ADL. Loss of sleep or poor sleep can directly limit ADL independence and also amplify other deficits. In acute and post-acute care, this must be considered in two ways. First, the timing of functional assessments should ideally attempt to be scheduled at times of optimal wakefulness. Moreover, interventions should be put into place to limit, to the extent deemed possible, poor sleep and its repercussions on everyday activities. 


\section{References}

Acheson, A., Richards, J. B., \& de Wit, H. (2007). Effects of sleep deprivation on impulsive behaviors in men and women. Physiol Behav, 91(5), 579-587.

Albouy, G., Fogel, S., Pottiez, H., Nguyen, V. A., Ray, L., Lungu, O. et al.. (2013). Daytime sleep enhances consolidation of the spatial but not motoric representation of motor sequence memory. PLoS One, 8(1), e52805.

Alvarez, J. A., \& Emory, E. (2006). Executive function and the frontal lobes: a meta-analytic review. Neuropsychol Rev, 16(1), 17-42.

American Psychiatric Association. (2013). Diagnostic and Statistical Manual of Mental Disorders Fifth Edition (DSM-5). Arlington, VA: American Psychiatric Publishing.

Atienza, M., Cantero, J. L., \& Stickgold, R. (2004). Posttraining sleep enhances automaticity in perceptual discrimination. $J$ Cogn Neurosci, 16(1), 53-64.

Australian Institute of Health and Welfare. (2007). Disability in Australia: acquired brain injury. Bulletin no.55. Canberra: AIHW.

Banhiran, W., Assanasen, P., Metheetrairut, C., Nopmaneejumruslers, C., Chotinaiwattarakul, W., \& Kerdnoppakhun, J. (2012). Functional outcomes of sleep in Thai patients with obstructive sleep-disordered breathing. Sleep Breath, 16(3), 663-675.

Baumann, C. R., Stocker, R., Imhof, H. G., Trentz, O., Hersberger, M., Mignot, E., \& Bassetti, C. L. (2005). Hypocretin-1 (orexin A) deficiency in acute traumatic brain injury. Neurology, 65(1), 147-149.

Baumann, C. R., Werth, E., Stocker, R., Ludwig, S., \& Bassetti, C. L. (2007). Sleep-wake disturbances 6 months after traumatic brain injury: a prospective study. Brain, 130(Pt 7), 18731883 .

Bedard, M. A., Montplaisir, J., Malo, J., Richer, F., \& Rouleau, I. (1993). Persistent neuropsychological deficits and vigilance impairment in sleep apnea syndrome after treatment with continuous positive airways pressure (CPAP). J Clin Exp Neuropsychol, 15(2), 330-341.

Bedard, M. A., Montplaisir, J., Richer, F., Rouleau, I., \& Malo, J. (1991). Obstructive sleep apnea syndrome: pathogenesis of neuropsychological deficits. J Clin Exp Neuropsychol, 13(6), 950-964. 
Beecroft, Jaime M., Ward, Michael, Younes, Magdy, Crombach, Shelley, Smith, Orla, \& Hanly, Patrick J. (2008). Sleep monitoring in the intensive care unit: comparison of nurse assessment, actigraphy and polysomnography. Intensive Care Medicine, 34(11), 2076-2083.

Bolge, S. C., Doan, J. F., Kannan, H., \& Baran, R. W. (2009). Association of insomnia with quality of life, work productivity, and activity impairment. Qual Life Res, 18(4), 415-422.

Borbely, A. A., Mattmann, P., Loepfe, M., Strauch, I., \& Lehmann, D. (1985). Effect of benzodiazepine hypnotics on all-night sleep EEG spectra. Hum Neurobiol, 4(3), 189-194.

Bourne, R. S., \& Mills, G. H. (2004). Sleep disruption in critically ill patients--pharmacological considerations. Anaesthesia, 59(4), 374-384.

Breed, S. T., Flanagan, S. R., \& Watson, K. R. (2004). The relationship between age and the self-report of health symptoms in persons with traumatic brain injury. Arch Phys Med Rehabil, 85(4 Suppl 2), S61-67.

Broughton, R., \& Baron, R. (1978). Sleep patterns in the intensive care unit and on the ward after acute myocardial infarction. Electroencephalogr Clin Neurophysiol, 45(3), 348-360.

Bryant, R. A., Marosszeky, J. E., Crooks, J., \& Gurka, J. A. (2000). Posttraumatic stress disorder after severe traumatic brain injury. Am J Psychiatry, 157(4), 629-631.

Buysse, D. J., Reynolds, C. F., 3rd, Monk, T. H., Berman, S. R., \& Kupfer, D. J. (1989). The Pittsburgh Sleep Quality Index: a new instrument for psychiatric practice and research. Psychiatry Res, 28(2), 193-213.

Bushnik, T., Englander, J., \& Katznelson, L. (2007). Fatigue after TBI: association with neuroendocrine abnormalities. Brain Inj, 21(6), 559-566.

Colarusso, C.P., \& Hammill, D.D. (2003). The Motor Free Visual Perception Test. Navato, CA: Academic Therapy Publications.

Carmona-Bernal, C., Ruiz-Garcia, A., Villa-Gil, M., Sanchez-Armengol, A., Quintana-Gallego, E., Ortega-Ruiz, F., et al. (2008). Quality of life in patients with congestive heart failure and central sleep apnea. Sleep Med, 9(6), 646-651.

Carrier, J., Monk, T. H., Buysse, D. J., \& Kupfer, D. J. (1997). Sleep and morningnesseveningness in the 'middle' years of life (20-59 y). J Sleep Res, 6(4), 230-237. 
Carskadon, M.A., \& Dement, W.C. (2011). Normal Human Sleep: an Overview. In M. Kryger, T. Roth \& W. Dement (Eds.), Principles and Practice of Sleep Medicine, 5th edition (pp. 16-26). St. Louis, MO: Elsevier.

Chasens, E. R., Sereika, S. M., Houze, M. P., \& Strollo, P. J. (2011). Subjective and objective appraisal of activity in adults with obstructive sleep apnea. J Aging Res, 2011, 751819.

Chee, M. W., \& Choo, W. C. (2004). Functional imaging of working memory after $24 \mathrm{hr}$ of total sleep deprivation. J Neurosci, 24(19), 4560-4567.

Choo, W. C., Lee, W. W., Venkatraman, V., Sheu, F. S., \& Chee, M. W. (2005). Dissociation of cortical regions modulated by both working memory load and sleep deprivation and by sleep deprivation alone. Neuroimage, 25(2), 579-587.

Cohen, M., Oksenberg, A., Snir, D., Stern, M. J., \& Groswasser, Z. (1992). Temporally related changes of sleep complaints in traumatic brain injured patients. J Neurol Neurosurg Psychiatry, 55(4), 313-315.

Cooper, A. B., Thornley, K. S., Young, G. B., Slutsky, A. S., Stewart, T. E., \& Hanly, P. J. (2000). Sleep in critically ill patients requiring mechanical ventilation.[Erratum appears in Chest 2001 Mar;119(3):993]. Chest, 117(3), 809-818.

Cronin, A. J., Keifer, J. C., Davies, M. F., King, T. S., \& Bixler, E. O. (2001). Postoperative sleep disturbance: influences of opioids and pain in humans. Sleep, 24(1), 39-44.

Curcio, G., Ferrara, M., \& De Gennaro, L. (2006). Sleep loss, learning capacity and academic performance. Sleep Med Rev, 10(5), 323-337.

Czeisler, C. A., \& Gooley, J. J. (2007). Sleep and circadian rhythms in humans. Cold Spring Harbor Symposia on Quantitative Biology, 72, 579-597.

Diekelmann, S., \& Born, J. (2010). The memory function of sleep. Nat Rev Neurosci, 11(2), 114126.

Dinges, D.F. (1992). Probing the limits of functional capability: the effects of sleep loss on shortduration tasks In R. J. Broughton \& R. D. Ogilvie (Eds.), Sleep, Arousal, and Performance (pp. 177-188). Boston, MA: Birkhauser.

Duclos, C., Dumont, M., Blais, H., Paquet, J., Laflamme, E., de Beaumont, L., et al. (2013). Rest-Activity Cycle Disturbances in the Acute Phase of Moderate to Severe Traumatic Brain Injury. Neurorehabil Neural Repair(Epub ahead of print). 
Dumont, M., \& Beaulieu, C. (2007). Light exposure in the natural environment: relevance to mood and sleep disorders. Sleep Med, 8(6), 557-565.

Fafrowicz, M., Oginska, H., Mojsa-Kaja, J., Marek, T., Golonka, K., \& Tucholska, K. (2010). Chronic sleep deficit and performance of a sustained attention task--an electrooculography study. Chronobiol Int, 27(5), 934-944.

Fenn, K. M., Nusbaum, H. C., \& Margoliash, D. (2003). Consolidation during sleep of perceptual learning of spoken language. Nature, 425(6958), 614-616.

Fischer, S., Hallschmid, M., Elsner, A. L., \& Born, J. (2002). Sleep forms memory for finger skills. Proc Natl Acad Sci U S A, 99(18), 11987-11991.

Foley, D., Ancoli-Israel, S., Britz, P., \& Walsh, J. (2004). Sleep disturbances and chronic disease in older adults: results of the 2003 National Sleep Foundation Sleep in America Survey. $J$ Psychosom Res, 56(5), 497-502.

Folstein, M.F., Folstein, S.E., \& McHugh, P.R. (1975). "Mini-mental state". A practical method for grading the cognitive state of patients for the clinician. Journal of Psychiatric Research, 12(3), 189-198.

Fontaine, D. K. (1989). Measurement of nocturnal sleep patterns in trauma patients. Heart Lung, 18(4), 402-410.

Fortier-Brochu, E., Beaulieu-Bonneau, S., Ivers, H., \& Morin, C. M. (2012). Insomnia and daytime cognitive performance: a meta-analysis. Sleep Med Rev, 16(1), 83-94.

Freedman, N. S., Gazendam, J., Levan, L., Pack, A. I., \& Schwab, R. J. (2001). Abnormal sleep/wake cycles and the effect of environmental noise on sleep disruption in the intensive care unit. Am J Respir Crit Care Med, 163(2), 451-457.

Freedman, N. S., Kotzer, N., \& Schwab, R. J. (1999). Patient perception of sleep quality and etiology of sleep disruption in the intensive care unit. Am J Respir Crit Care Med, 159(4 Pt 1), 1155-1162.

Friese RS, Diaz-Arrastia R, McBride D, Frankel H, Gentilello LM. (2007). Quantity and quality of sleep in the surgical intensive care unit: are our patients sleeping? J Trauma, 63(6), 12101214.

Frohnhofen, H., Popp, R., Frohnhofen, K., \& Fulda, S. (2013). Impact of daytime sleepiness on rehabilitation outcome in the elderly. Adv Exp Med Biol, 755, 103-110. 
Gabor, J.Y., Cooper, A.B., Crombach, S.A., Lee, B., Kadikar, N., Bettger, H.E., \& Hanly, P.J. (2003). Contribution of the intensive care unit environment to sleep disruption in mechanically ventilated patients and healthy subjects. Am J Respir Crti Care Med, 167(5), 708-715.

Gehlbach, Brian K., Chapotot, Florian, Leproult, Rachel, Whitmore, Harry, Poston, Jason, Pohlman, Mark, . . . Van Cauter, Eve. (2012). Temporal disorganization of circadian rhythmicity and sleep-wake regulation in mechanically ventilated patients receiving continuous intravenous sedation. Sleep, 35(8), 1105-1114.

Geneau, D., \& Taillefer, D. (1996). Le "Protocole d'Examen Cognitif de la Personne Âgée Version Révisée" (PECPA-2r): Normalisation par groupes d'âge et antécédents éducationnels chez des sujets québécois francophones. Paper presented at the Allocution présentée au 2ième Colloque de Psychogériatrie du C.C.F.P., St-Hyacinthe, Québec.

Germain, A. (2013). Sleep disturbances as the hallmark of PTSD: where are we now? Am $J$ Psychiatry, 170(4), 372-382.

Gimenez, S., Clos, S., Romero, S., Grasa, E., Morte, A., \& Barbanoj, M. J. (2007). Effects of olanzapine, risperidone and haloperidol on sleep after a single oral morning dose in healthy volunteers. Psychopharmacology (Berl), 190(4), 507-516.

Goel, N., Rao, H., Durmer, J.S., \& Dinges, D.F. (2009). Neurocognitive consequences of sleep deprivation. Seminars in Neurology, 29(4), 320-339.

Harrison, Y., \& Horne, J. A. (2000). The impact of sleep deprivation on decision making: a review. J Exp Psychol Appl, 6(3), 236-249.

Iber, C, Ancoli-Israel, S, \& Quan, S.F,, for the American Academy of Sleep Medicine. (2007). The AASM manual for the scoring of sleep and associated events: rules, terminology, and technical specifications. Westchester, IL: Amercian Academy of Sleep Medicine.

Jones, K., \& Harrison, Y. (2001). Frontal lobe function, sleep loss and fragmented sleep. Sleep Med Rev, 5(6), 463-475.

Kavey, N. B., \& Ahshuler, K. Z. (1979). Sleep in herniorrhaphy patients. Am J Surg, 138(5), 683-687.

Killgore, W. D. (2010). Effects of sleep deprivation on cognition. Prog Brain Res, 185, 105-129.

Langlois-Orman, J.A., Kraus, J.F., Zaloshnja, E., \& Miller, T. (2011). Epidemiology. In J. M. Silver, T. W. McAllister \& S. C. Yudofsky (Eds.), Textbook of Traumatic Brain Injury (2nd ed., pp. 3-22). Washington DC: American Psychiatric Publishing. 
Lee, M., Choh, A. C., Demerath, E. W., Knutson, K. L., Duren, D. L., Sherwood, R. J., et al. (2009). Sleep disturbance in relation to health-related quality of life in adults: the Fels Longitudinal Study. J Nutr Health Aging, 13(6), 576-583.

Le Guen, M., Nicolas-Robin, A., Lebard, C., Arnulf, I., \& Langeron, O. (2014). Earplugs and eye masks vs routine care prevent sleep impairment in post-anaesthesia care unit: a randomized study. Br J Anaesth, 112(1), 89-95.

Lezak, M.D., Howieson, D.B., \& Loring, D.W. (2004). Neuropsychological Assessment (4th ed.). New York, NY: Oxford University Press.

Lim, J., \& Dinges, D. F. (2008). Sleep deprivation and vigilant attention. Ann N Y Acad Sci, $1129,305-322$.

Lovato, N., \& Lack, L. (2010). The effects of napping on cognitive functioning. Prog Brain Res, $185,155-166$.

Makley, M. J., English, J. B., Drubach, D. A., Kreuz, A. J., Celnik, P. A., \& Tarwater, P. M. (2008). Prevalence of sleep disturbance in closed head injury patients in a rehabilitation unit. Neurorehabilitation \& Neural Repair, 22(4), 341-347.

Makley, M.J., Johnson-Greene, L.T., Patrick M., Kreuz, A.J., Spiro, J., Rao, V., \& Celnik, Pablo A. (2009). Return of memory and sleep efficiency following moderate to severe closed head injury. Neurorehabilitation \& Neural Repair, 23(4), 320-326.

Manber, R., Carney, C., Edinger, J., Epstein, D., Friedman, L., Haynes, P. L., et al. (2012). Dissemination of CBTI to the non-sleep specialist: protocol development and training issues. $J$ Clin Sleep Med, 8(2), 209-218.

Mermigkis, C., Bouloukaki, I., Antoniou, K. M., Mermigkis, D., Psathakis, K., Giannarakis, I., et al. (2013). CPAP therapy in patients with idiopathic pulmonary fibrosis and obstructive sleep apnea: does it offer a better quality of life and sleep? Sleep Breath, 17(4), 1137-1143.

Mindell, J. A., Owens, J. A., \& Carskadon, M. A. (1999). Developmental features of sleep. Child Adolesc Psychiatr Clin N Am, 8(4), 695-725.

Moore, R. Y. (1997). Circadian rhythms: basic neurobiology and clinical applications. Annu Rev Med, 48, 253-266.

Morin, C.M. (1993). Insomnia, psychological Assessment and Management: The Guilford Press. 
Morin, C. M. (2004). Cognitive-behavioral approaches to the treatment of insomnia. J Clin Psychiatry, 65 Suppl 16, 33-40.

Mu, Q., Nahas, Z., Johnson, K. A., Yamanaka, K., Mishory, A., Koola, J., et al. (2005). Decreased cortical response to verbal working memory following sleep deprivation. Sleep, 28(1), 55-67.

Munch, M., \& Bromundt, V. (2012). Light and chronobiology: implications for health and disease. Dialogues Clin Neurosci, 14(4), 448-453.

Nakase-Richardson, R., Sherer, M., Barnett, S.D., Yablon, S.A., Evans, C.C., Kretzmer, T., et al. (2013). Prospective evaluation of the nature, course, and impact of acute sleep abnormality after traumatic brain injury. Arch Phys Med Rehabil, 94(5), 875-882.

Nelson, J. E., Meier, D. E., Oei, E. J., Nierman, D. M., Senzel, R. S., Manfredi, P. L., et al. (2001). Self-reported symptom experience of critically ill cancer patients receiving intensive care. Crit Care Med, 29(2), 277-282.

Ohayon, M. M., Carskadon, M. A., Guilleminault, C., \& Vitiello, M. V. (2004). Meta-analysis of quantitative sleep parameters from childhood to old age in healthy individuals: developing normative sleep values across the human lifespan. Sleep, 27(7), 1255-1273.

Olsen, H. W., Lawrence, W. A., Bottarini, G., \& Pises, P. (1977). The fiberoptic approach to dilation of stenotic lesions of the esophagus. Gastrointest Endosc, 23(4), 201-202.

Ouellet, M. C., Beaulieu-Bonneau, S., \& Morin, C. M. (2006). Insomnia in patients with traumatic brain injury: frequency, characteristics, and risk factors. J Head Trauma Rehabil, 21(3), 199-212.

Ouellet, M. C., \& Morin, C. M. (2004). Cognitive behavioral therapy for insomnia associated with traumatic brain injury: a single-case study. Arch Phys Med Rehabil, 85(8), 1298-1302.

Ouellet, M. C., \& Morin, C. M. (2007). Efficacy of cognitive-behavioral therapy for insomnia associated with traumatic brain injury: a single-case experimental design. Arch Phys Med Rehabil, 88(12), 1581-1592.

Parcell, D.L., Ponsford, J.L., Rajaratnam, S.M., \& Redman, J.R. (2006). Self-reported changes to nighttime sleep after traumatic brain injury. Archives of Physical Medicine \& Rehabilitation, $87(2), 278-285$. 
Persson Waye, K., Elmenhorst, E. M., Croy, I., \& Pedersen, E. (2013). Improvement of intensive care unit sound environment and analyses of consequences on sleep: an experimental study. Sleep Med, 14(12), 1334-1340.

Rao V, Spiro J, Vaishnavi S, Rastogi P, Mielke M, Noll K, Cornwell E, Schretlen D, Makley M. (2008). Prevalence and types of sleep disturbances acutely after traumatic brain injury. Brain Injury, 22(5), 381-386.

Rappaport, M., Hall, K. M., Hopkins, K., Belleza, T., \& Cope, D. N. (1982). Disability rating scale for severe head trauma: coma to community. Archives of Physical Medicine \& Rehabilitation, 63(3), 118-123.

Reynolds, A. C., \& Banks, S. (2010). Total sleep deprivation, chronic sleep restriction and sleep disruption. Prog Brain Res, 185, 91-103.

Richards, K. C., \& Bairnsfather, L. (1988). A description of night sleep patterns in the critical care unit. Heart Lung, 17(1), 35-42.

Richardson, A., Allsop, M., Coghill, E., \& Turnock, C. (2007). Earplugs and eye masks: do they improve critical care patients' sleep? Nurs Crit Care, 12(6), 278-286.

Riegel, B., Ratcliffe, S. J., Weintraub, W. S., Sayers, S. L., Goldberg, L. R., Potashnik, S., et al. (2012). Double jeopardy: the influence of excessive daytime sleepiness and impaired cognition on health-related quality of life in adults with heart failure. Eur J Heart Fail, 14(7), 730-736.

Riemersma-van der Lek, R. F., Swaab, D. F., Twisk, J., Hol, E. M., Hoogendijk, W. J., \& Van Someren, E. J. (2008). Effect of bright light and melatonin on cognitive and noncognitive function in elderly residents of group care facilities: a randomized controlled trial. JAMA, 299(22), 2642-2655.

Roozenbeek, B., Maas, A. I., \& Menon, D. K. (2013). Changing patterns in the epidemiology of traumatic brain injury. Nat Rev Neurol, 9(4), 231-236.

Sagaspe, P., Charles, A., Taillard, J., Bioulac, B., \& Philip, P. (2003). [Inhibition and working memory: effect of acute sleep deprivation on a random letter generation task]. Can J Exp Psychol, 57(4), 265-273.

Simini, B. (1999). Patients' perceptions of intensive care. Lancet, 354(9178), 571-572.

Sinclair, K. L., Ponsford, J. L., Taffe, J., Lockley, S. W., \& Rajaratnam, S. M. (2013).

Randomized Controlled Trial of Light Therapy for Fatigue Following Traumatic Brain Injury. Neurorehabil Neural Repair (Epub ahead of print). 
Shilo, L., Dagan, Y., Smorjik, Y., Weinberg, U., Dolev, S., Komptel, B. et al.. (1999). Patients in the intensive care unit suffer from severe lack of sleep associated with loss of normal melatonin secretion pattern. Am J Med Sci, 317(5), 278-281.

Sloane, P. D., Williams, C. S., Mitchell, C. M., Preisser, J. S., Wood, W., Barrick, A. L., et al. (2007). High-intensity environmental light in dementia: effect on sleep and activity. $J \mathrm{Am}$ Geriatr Soc, 55(10), 1524-1533.

Smith, C. (1996). Sleep states, memory processes and synaptic plasticity. Behav Brain Res, $78(1), 49-56$.

Sohlberg, M. M., \& Mateer, C. A. (1987). Effectiveness of an attention-training program. J Clin Exp Neuropsychol, 9(2), 117-130.

Spira, A. P., Covinsky, K., Rebok, G. W., Punjabi, N. M., Stone, K. L., Hillier, T. A., et al. (2012). Poor sleep quality and functional decline in older women. J Am Geriatr Soc, 60(6), 1092-1098.

Stickgold, R., James, L., \& Hobson, J. A. (2000). Visual discrimination learning requires sleep after training. Nat Neurosci, 3(12), 1237-1238.

Su, C. S., Liu, K. T., Panjapornpon, K., Andrews, N., \& Foldvary-Schaefer, N. (2012). Functional outcomes in patients with REM-related obstructive sleep apnea treated with positive airway pressure therapy. J Clin Sleep Med, 8(3), 243-247.

Tagliaferri, F., Compagnone, C., Korsic, M., Servadei, F., \& Kraus, J. (2006). A systematic review of brain injury epidemiology in Europe. Acta Neurochir (Wien), 148(3), 255-268;

discussion 268.

Takahashi, J. S., \& Zatz, M. (1982). Regulation of circadian rhythmicity. Science, 217(4565), 1104-1111.

Tamburri, L. M., DiBrienza, R., Zozula, R., \& Redeker, N. S. (2004). Nocturnal care interactions with patients in critical care units. Am J Crit Care, 13(2), 102-112; quiz 114-105.

Teixeira, V. G., Faccenda, J. F., \& Douglas, N. J. (2004). Functional status in patients with narcolepsy. Sleep Med, 5(5), 477-483. 
Tomasi, D., Wang, R. L., Telang, F., Boronikolas, V., Jayne, M. C., Wang, G. J., et al. (2009). Impairment of attentional networks after 1 night of sleep deprivation. Cereb Cortex, 19(1), 233240.

Topf, M. (1992). Effects of personal control over hospital noise on sleep. Res Nurs Health, 15(1), 19-28.

Topf, M., \& Davis, J. E. (1993). Critical care unit noise and rapid eye movement (REM) sleep. Heart Lung, 22(3), 252-258.

Trzepacz, P. T., Mittal, D., Torres, R., Kanary, K., Norton, J., \& Jimerson, N. (2001). Validation of the Delirium Rating Scale-revised-98: comparison with the delirium rating scale and the cognitive test for delirium. J Neuropsychiatry Clin Neurosci, 13(2), 229-242.

Van Cauter, E., Leproult, R., \& Plat, L. (2000). Age-related changes in slow wave sleep and REM sleep and relationship with growth hormone and cortisol levels in healthy men. JAMA, 284(7), 861-868.

Van Someren, E.J. Kessler, A., Mirmiran, M., \& Swaab, D.F. (1997). Indirect bright light improves circadian rest-activity rhythm disturbances in demented patients. Biological Psychiatry, 41, 955-963.

Walker, M. P. (2004). Issues surrounding sleep-dependent memory consolidation and plasticity. Cellular \& Molecular Life Sciences, 61(24), 3009-3015.

Walker, M. P., Brakefield, T., Morgan, A., Hobson, J. A., \& Stickgold, R. (2002). Practice with sleep makes perfect: sleep-dependent motor skill learning. Neuron, 35(1), 205-211.

Walker, M. P., \& Stickgold, R. (2006). Sleep, memory, and plasticity. Annu Rev Psychol, 57, 139-166.

Walker, M. P., Stickgold, R., Alsop, D., Gaab, N., \& Schlaug, G. (2005). Sleep-dependent motor memory plasticity in the human brain. Neuroscience, 133(4), 911-917.

Wallace, C. J., Robins, J., Alvord, L. S., \& Walker, J. M. (1999). The effect of earplugs on sleep measures during exposure to simulated intensive care unit noise. Am J Crit Care, 8(4), 210-219.

Weaver, T. E., Laizner, A. M., Evans, L. K., Maislin, G., Chugh, D. K., Lyon, K., et al. (1997). An instrument to measure functional status outcomes for disorders of excessive sleepiness. Sleep, 20(10), 835-843. 
Welsh, M.C., \& Pennington, B.F. (1988). Assessing frontal lobe functioning in children: views from developmental psychology. Developmental Neuropsychology, 4(3), 199-230.

Welsh, M.C., Pennington, B.F., \& Groisser, D.B. (1991). A normative-developmental study of executive function: A window on prefrontal function in children. Developmental Neuropsychology, 7(2), 131-149.

Wilson, M. A. (2002). Hippocampal memory formation, plasticity, and the role of sleep. Neurobiol Learn Mem, 78(3), 565-569.

Wilson, S., \& Argyropoulos, S. (2005). Antidepressants and sleep: a qualitative review of the literature. Drugs, 65(7), 927-947.

Worthington, A. D., \& Melia, Y. (2006). Rehabilitation is compromised by arousal and sleep disorders: results of a survey of rehabilitation centres. Brain Inj, 20(3), 327-332.

Yetkin, S., Aydin, H., \& Ozgen, F. (2010). Polysomnography in patients with post-traumatic stress disorder. Psychiatry Clin Neurosci, 64(3), 309-317.

Yoo, S. S., Hu, P. T., Gujar, N., Jolesz, F. A., \& Walker, M. P. (2007). A deficit in the ability to form new human memories without sleep. Nat Neurosci, 10(3), 385-392.

Zelazo, P.D., \& Müller, U. (2002). Executive Function in Typical and Atypical Development. In U. Goswani (Ed.), Blackwell Handbook of Childhood Cognitive Development (pp. 445-469). Oxford: Blackwell.

Zhang, J., Lamers, F., Hickie, I. B., He, J. P., Feig, E., \& Merikangas, K. R. (2013). Differentiating nonrestorative sleep from nocturnal insomnia symptoms: demographic, clinical, inflammatory, and functional correlates. Sleep, 36(5), 671-679. 


\section{Figure 1. Sleep architecture of a healthy adult subject}

Figure 1 depicts the sleep architecture of a healthy adult subject. Sleep occurs in consecutive cycles of approximately 90 minutes, characterised by varying proportions of N1, N2, N3, and rapid eye movement (REM) sleep. Stage N3, or deep sleep, occurs mainly in the first sleep cycles, diminishing in proportion as the sleep period advances. Conversely, the proportion of REM sleep increases throughout the night, and is mainly present during later sleep cycles.

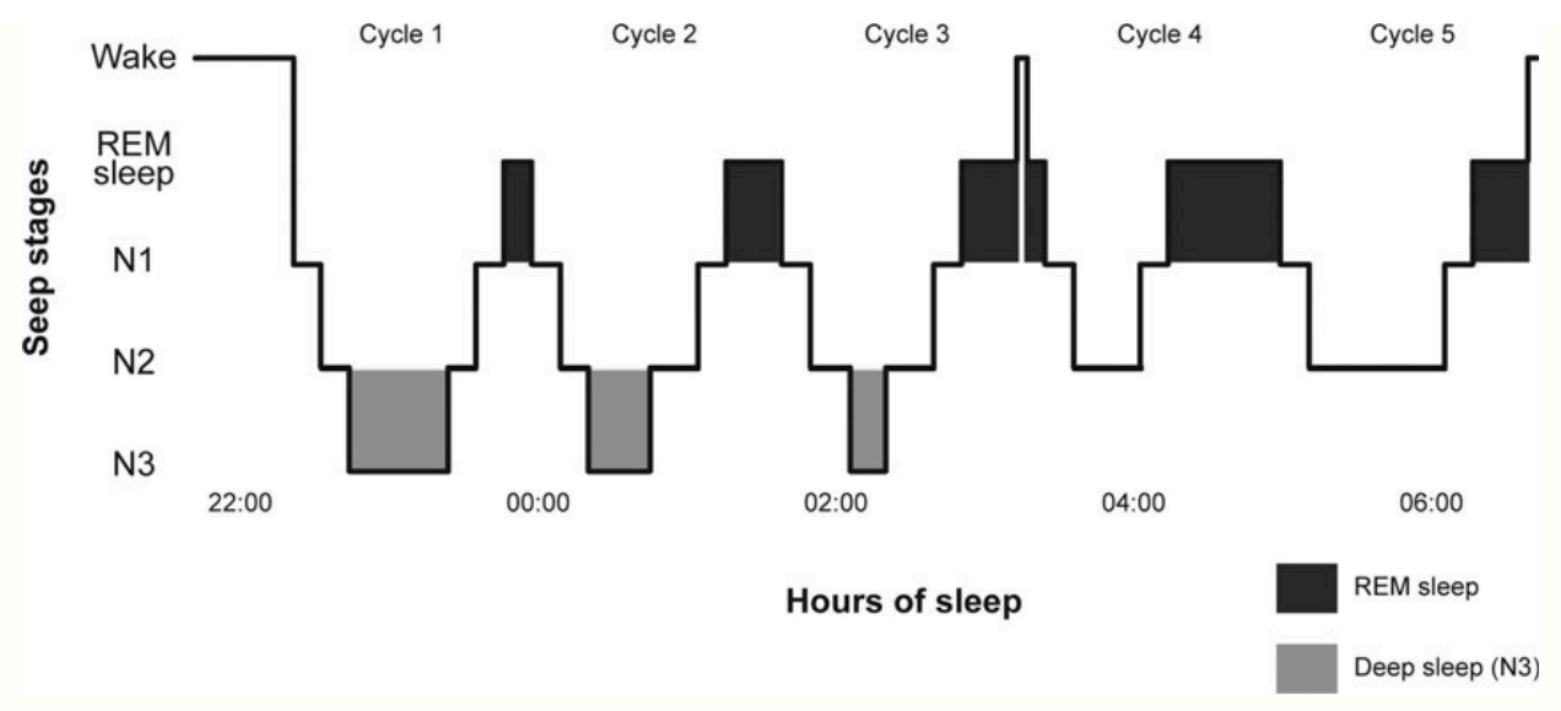

